\title{
La discriminación laboral en razón del género y la edad en Colombia
}

\section{Labor discrimination based on gender and age in Colombia}

\author{
José Javier Nuvaez Castillo \\ jose.nuvaezc@campusucc.edu.co \\ Universidad Cooperativa de Colombia \\ Santa Marta/UCCIDERGRUP \\ Colombia \\ https://orcid.org/0000-0002-0974-3738
}

Recibido: 19 de septiembre del 2018

Aprobado: 28 de noviembre del 2018

\begin{abstract}
RESUMEN
La discriminación o diferenciación laboral se define como un trato diferenciado basado en razones diversas; es el señalamiento prejuicioso a una determinada persona con ciertas características, adjudicándole una condición o sentido de inferioridad. En tal sentido, con fines de análisis se examina este concepto, para indagar su alcance 0 trascendencia; en un estudio enmarcado bajo un referente teórico basado en el criterio de expertos en el área. El estudio es una investigación documental con un diseño bibliográfico. Los resultados reflejan una problemática actual, que genera un impacto socio-cultural en Colombia, producido por lo alarmante en los niveles de discriminación en el país, debiendo concluir que en la medida en que se perpetúe este panorama desolador en materia laboral, el trabajo continuará siendo un motivo de frustración y no una fuente de realización personal de los trabajadores colombianos, en razón del rechazo, la exclusión a lo que son sujetos los mismos.
\end{abstract}

Descriptores: Discriminación; discriminación por edad; especie humana; derecho laboral; trabajo

\begin{abstract}
Labor discrimination or differentiation is defined as differentiated treatment based on different reasons; It is the prejudicial indication to a certain person with certain characteristics, awarding him a condition or sense of inferiority. In this sense, for the purpose of analysis this concept is examined, to investigate its scope or transcendence; in a study framed under a theoretical reference based on the criteria of experts in the area. The study is a documentary research with a bibliographic design. The results reflect a current problem, which generates a socio-cultural impact in Colombia,
\end{abstract}


produced by the alarming levels of discrimination in the country, and must conclude that to the extent that this devastating panorama in labor matters is perpetuated, the work will continue being a source of frustration and not a source of personal fulfillment for Colombian workers, because of the rejection, the exclusion to which they are subject.

Descriptors: Discrimination; age discrimination; human species; Labour law; Labour.

\section{INTRODUCCION}

La discriminación laboral suele presentarse en todas las latitudes mundiales, como una forma de segregar o excluir a un conglomerado humano que forma parte de una población, entidad, organización, sea pública o privada. No obstante, los legisladores de innumerables países han intentado crear normativas que propicien la eliminación o minimización de esa pretendida distinción en el sitio de trabajo.

Colombia no es la excepción, pero aun adoptando diferentes convenios internacionales y promulgando normatividad respecto al tema objeto de estudio, cada vez es mayor la vulneración a los derechos fundamentales laborales por razones de género y edad. En este sentido, se observan diferenciaciones que dificultan la calidad de vida de los trabajadores, debiendo en las empresas, por el contrario, promover la protección especial de los derechos que tienen los ciudadanos a ser tratados en condiciones de igualdad, sin que puedan ser discriminados en razón de su condición de vida para acceder al trabajo.

De allí que esta investigación tenga como objeto de estudio el análisis de la discriminación laboral por razón del género y la edad en Colombia, generando un impacto socio jurídico en la medida en que presenta alternativas que contribuyen a contrarrestar el problema de la discriminación en los casos objeto de estudio, todo lo cual se realiza bajo un referente teórico y metodológico de análisis e interpretación preciso.

Es así como, de cara al fenómeno de la adhesión a la internalización de las normas laborales y la consiguiente constitucionalización del derecho laboral en el estado colombiano, este se comprometió a adoptar las medidas necesarias para garantizar las 
posibilidades de acceso de hombres, mujeres, jóvenes, niños, niñas y personas mayores, a un trabajo digno en condiciones de igualdad; sin embargo, pese a la protección especial o no que merecen los sujetos en mención, la realidad laboral indica que cada vez es mayor la vulneración a los derechos fundamentales laborales de los mismos.

De allí que esta discriminación laboral suele presentarse en todos los hemisferios y Estados, sean estos desarrollados o subdesarrollados, también sean estos de cultura liberal o no. Es así que través de los convenios y recomendaciones suscritas por los estados miembros ante la Organización Internacional del Trabajo (OIT, 2014),

Todo Estado que ratifique el Convenio se obliga a formular y llevar a cabo una política nacional que promueva la igualdad de oportunidades y de trato, con objeto de eliminar cualquier discriminación en los siguientes terrenos: 1 . Acceso a la formación profesional; 2. Admisión en el empleo y en las diversas ocupaciones; 3 . Condiciones de trabajo. (p. 35)

Asimismo, la diagnosis situacional en este estudio permitió documentar discriminación de género como devenidas de aquellas normas, decisiones y prácticas que no permiten la igualdad los intereses y derechos de varones y mujeres, y/o que dan lugar a resultados de desigualdad derivado del trato desconsiderado. De la misma manera, se da la discriminación por edad cuando se limita o se impide a un individuo a la ejecución de una labor remunerada por sus condiciones etarias, ya por ser demasiado joven o ser un adulto mayor, sin permitirle en tal sentido el derecho al trabajo consagrado en diversas legislaciones del mundo.

En tal sentido, con miras a debilitar tal situación de injusticia laboral, en razón de la vulneración a ciertas condiciones de trabajo del ciudadano, en Colombia se han venido expidiendo normativas diversas, con el ánimo de promover la protección especial de los derechos que tienen los ciudadanos a ser tratados en condiciones de igualdad, sin que puedan ser discriminados en razón de su género o edad para acceder al trabajo. Lo anterior conduce a deducir que ningún empleador tiene el derecho de eliminar a un aspirante al trabajo por el solo hecho de su identidad o edad, a no ser que condiciones 
específicas exijan tal situación; no obstante, se observan en algunos escenarios laborales, transgresiones frecuentes a tales condiciones personales y sociales.

En este contexto, se percibe la distribución desproporcionada de mujeres y varones por sectores laborales específicos segregación horizontal lo cual se observa como un hecho constatable, que se evidencia a través de la calificación de masculino o femenino en tanto características atribuidas a bastantes trabajos. Ejemplos de estas situaciones se han venido observando, socialmente, en la carrera de magisterio, y en particular en la educación infantil, lo cual pareciera considerarse como un trabajo femenino, mientras que las actividades de ingeniería en obras públicas suelen etiquetarse como masculinas, por el supuesto trabajo forzoso o riesgoso en este caso.

Por otra parte, se observan síntomas de una problemática en la realidad organizacional de Colombia al no equiparar hombres y mujeres en cargos públicos o privados, por supuesta ineficacia de la mujer, siendo en algunos casos notoria una desigualdad en cuanto a la remuneración recibida o al trato prestado, siendo, en su mayoría, tales mujeres las más afectadas.

Asimismo, pareciera observarse, de acuerdo a la reflexividad y percepción del investigador, que las situaciones de discriminación por vejez se presentan, en principio, cuando se llega a los límites establecidos para la tercera edad, o cuando la persona tiene una edad avanzada. A partir de entonces, es común que tenga dificultades por esa razón para conseguir otro empleo y que peligre su subsistencia por no tener la edad para jubilarse.

Esta situación desventajosa en la praxis empresarial pública y privada ocurre porque, salvo situaciones de reconocida capacidad o antecedentes notorios, la tendencia generalizada es cubrir las plazas vacantes con trabajadores más jóvenes, con una presunción muchas veces fallida, de que tal elemento influye en una mayor productividad. Aparece otro abuso que es frecuente, el tomar estas personas para trabajos transitorios o tareas de difícil aceptación por otros, los cuales se aceptan porque no tienen otra posibilidad para satisfacer sus necesidades, más aún cuando 
tienen obligaciones familiares.

Sobre el particular, de entrevistas informales suscitadas en el ,ámbito laboral con antelación a este estudio, se evidencian manifestaciones de trabajadores 0 empleadores acerca de las causas que originan la problemática planteada, lo cual pareciera derivar del desconocimiento de asalariados de su derecho al trabajo en condiciones óptimas, además de la intimidación de perder su empleo, que hoy en día se encuentra tan escaso en el país a pesar de que las cifras muestran lo contrario; originando como consecuencia, distinciones, exclusiones, preferencias en el empleo, además de la ocupación de mujeres y personas mayores en cargos inclusive indignos, considerados como actos segregacionistas, separatistas, e incluso prejuiciosos en el ámbito laboral.

Con ello se impide, como consecuencia, los ascensos en el puesto de trabajo desempeñado, la conservación del empleo o las condiciones de trabajo adecuadas que deben prevalecer para los trabajadores. En tal virtud, el estudio trata lo concerniente al análisis de la discriminación laboral por razones de género y edad en Colombia, siendo esta investigación desarrollada en el período comprendido entre enero de 2016 a junio de 2016. Desde lo planteado, la investigación gira en torno a la siguiente interrogante: ¿Cómo se desarrolla la discriminación laboral en razón del género y la edad en Colombia en el período comprendido entre enero de 2016 a junio de $2016 ?$

\section{OBJETIVO DE LA INVESTIGACIÓN}

Analizar la discriminación laboral en razón del género y la edad en Colombia en el período comprendido entre enero de 2016 a junio de 2016.

\section{INVESTIGACIONES PREVIAS}

Se presentan diversas investigaciones que permiten estudiar el devenir del tema sobre la discriminación laboral en Colombia, constituyéndose en un aporte significativo para analizar el comportamiento de las variables de estudio, en contexto de estudio similar al 
planteado en la actual investigación, en este sentido, se presenta a (Vargas y Cortes, 2011), quienes en sus principales conclusiones arrojan que

Durante el desarrollo de la investigación y el procesamiento de las encuestas del DANE se observó el comportamiento de las variables que en el estudio son influyentes en la discriminación laboral por edad, y se concluye que la discriminación después de los 40 años está latente para la población en general, bien sea por falta de capacitación, bajos niveles de educación, lugar donde se habita y hasta el estrato socioeconómico, además se encuentra que a pesar de la marcada discriminación, la necesidad de devengar un ingreso mínimo de subsistencia obliga a gran parte de la población a mantenerse ocupados, ya sea como independientes, subempleados, 0 trabajadores informales. (p. 50)

En función de lo expuesto, se evidencia que existe un desinterés por parte de los empleadores de contratar a personas mayores a los 40 años de edad, generando así una brecha entre quienes pueden asumir productivamente una plaza de trabajo, pero por motivos de discriminación quedan a la deriva en el sector desempleo, esto implica pérdidas no solo desde el punto de vista social, sino económico para el país, por cuanto parte de su población activa queda al margen de producir por el hecho discriminatorio. Así mismo, (Barraza Narváez, 2011), plantea que

La discriminación salarial y la segregación laboral de género han disminuido con el tiempo pero aún persisten; para analizar la existencia de estos problemas en la región Caribe se utilizó como base de datos la Encuesta Continua de Hogares del año 2005, de la cual se extrajo información para las áreas metropolitanas de Barranquilla, Cartagena y Montería. Se encontró evidencia de segregación laboral de género en la posición ocupacional, tipo de ocupación y rama de actividad a la cual pertenece el individuo. Estimando ecuaciones mincerianas del ingreso y calculando la descomposición de Oaxaca se encontró evidencia de diferencias salariales entre hombres y mujeres, las cuales se explican en gran parte por la existencia de discriminación. (p. 5).

La discriminación por género, afecta en las diferencias salariales entre hombres y mujeres, generándose así una brecha entre ambos segmentos, promoviéndose así, la discriminación laboral en diversas dimensiones que evidencian la existencia aun de 
percepciones negativas entre los habitantes del país, lo cual implica la existencia de una cultura machista que perjudica las relaciones interpersonales y laborales en la sociedad colombiana.

En otro orden de ideas, la investigación de (Castillo Mayen, 2011), plantea la discriminación de género como un caldo de cultivo social en donde se afianzan los estereotipos machistas, en este sentido, plantea que

Se puede afirmar que las estrategias de marketing en las que se acentúan las características estereotípicas de las mujeres y de los hombres, los programas televisivos que se basan en hacer salientes estas categorías, o cualquier otro uso indiscriminado de estos estereotipos (ej., chistes, refranes) están fomentando el apoyo hacia la jerarquía grupal basada en el género, y por tanto, favoreciendo que los hombres continúen siendo el grupo dominante en este sistema y las mujeres el grupo subordinado. (p. 437)

El imaginario social sobre la imagen dominante del hombre es reforzado por estrategias de marketing que impregnan en el idealismo popular como una verdad que refuerza la conducta estereotipada sobre el rol sumiso que debe poseer la mujer en la sociedad, esto coadyuva progresivamente a fortalecer las creencias discriminatorias en el campo laboral a causa de la diferencia de género, es imprescindible generar conciencia sobre el papel preponderante que juegan los mitos creados en la cultura popular y que pueden ser banalizados en medios de comunicación social por el hecho de subir rating, cuando en el fondo perjudican la edificación de una sociedad basada en la igualdad, en el respeto mutuo, en la interculturalidad como medio para construir relaciones asertivas que eviten actos discriminatorios en lo social y laboral.

\section{REFERENCIAL TEÓRICO}

\section{Discriminación laboral}

La discriminación laboral puede ser generada por diversos factores que afectan el desarrollo asertivo de relaciones laborales entre el patrono y los empleados, en este sentido, la (OIT, 1960), define la Discriminación laboral como

Cualquier distinción, exclusión o preferencia basada en motivos de raza, 
color, sexo, religión, opinión política, ascendencia nacional, origen social o cualquier otro motivo especificado por un Estado parte en el Convenio, previa consulta con las organizaciones representativas de empleadores y de trabajadores, y con otros organismos apropiados y que tenga por efecto anular o alterar la igualdad de oportunidades o de trato en el empleo o en la ocupación. (p. 1)

Se destaca un problema multifactorial en el desarrollo de la discriminación laboral, por cuanto puede obedecer a intereses financieros del patrón, discriminación racial, política, religiosa, siendo estos elementos que son contrarios a establecer un clima de paz no solo en la empresa, sino en la sociedad, además que violan el derecho al trabajo que posee toda persona, generándose así, persecución y exclusión por motivos que no tienen que ver con el efectivo rendimiento de la persona en su puesto de trabajo, así como faltas a las normas o productividad propuesta por la empresa, de ese modo, se fomenta un clima de violencia. Cabe destacar que en cuanto a la edad, en Colombia se expide en el (Diario Oficial No. 45.777 de diciembre 30 de 2004, la ley 931), en donde se destaca,

ARTíCULO 10. OBJETIVO. La presente ley tiene por objeto la protección especial por parte del Estado de los derechos que tienen los ciudadanos a ser tratados en condiciones de igualdad, sin que puedan ser discriminados en razón de su edad para acceder al trabajo. ARTíCULO 2o. PROHIBICIÓN. Ninguna persona natural o jurídica, de derecho público o privado, podrá exigir a los aspirantes a ocupar un cargo o ejercer un trabajo, cumplir con un rango de edad determinado para ser tenido en cuenta en la decisión que defina la aprobación de su aspiración laboral. Los requisitos para acceder a un cargo que se encuentre vacante o a ejercer un trabajo deberán referirse a méritos o calidades de experiencia, profesión u ocupación. ( $\mathrm{p} 1)$

La ley prevé la protección de las personas en poder concursar a un empleo en igualdad de condiciones, solo siendo resaltadas para tal puesto, los propios méritos obtenidos mediante la formación y experiencia, constituyéndose en indicadores a medir a la hora de definir un determinado cargo, incurrir en penalizaciones por motivos ajenos a los planteados, es infringir no solo la ley, sino los tratados internacionales establecidos para evitar la discriminación laboral. Lo anterior conduce, en el criterio de Amaya (2013) a 
que ningún empleador tiene el derecho de eliminar a un aspirante al trabajo por el solo hecho de su edad.

En este contexto, las situaciones de discriminación por vejez se presentan, en principio, cuando la persona tiene una edad avanzada. A partir de entonces, es común que tenga dificultades por esa razón para conseguir otro empleo y que peligre su subsistencia por no tener la edad para jubilarse. Esto ocurre porque, salvo situaciones de reconocida capacidad o antecedentes notorios, la tendencia generalizada es cubrir las plazas vacantes con trabajadores más jóvenes, con una presunción muchas veces fallida, de que tal elemento influye en una mayor productividad.

Aparece también otro abuso que es frecuente, el tomar estas personas para trabajos transitorios o tareas de difícil aceptación por otros, que ellos aceptan porque no tienen otra posibilidad para satisfacer sus necesidades, más aún cuando tienen obligaciones familiares, según (Castro, 2010):

La discriminación laboral consiste en toda distinción, exclusión o preferencia de trato que, ocurrida con motivo o con ocasión de una relación de trabajo, se base en un criterio de raza, color, sexo, religión, sindicación, opinión política o cualquier otro que se considere irracional o injustificado, y que tenga por efecto alterar o anular la igualdad de trato en el empleo y la ocupación. (p. 8).

Complementando lo planteado, en Colombia existe una tendencia de rechazar a las personas al postularse a los puestos de trabajo por motivos de la edad, lo cual promueve el desempleo a pesar de que muchas de estas personas poseen las capacidades para ejercer efectivamente el trabajo. En este sentido, (Jiménez, 2015), plantea que:

Para nadie es un secreto el que los patronos rechazan a solicitantes de empleo que ofrecen madurez, estabilidad, experiencia y buenas calificaciones sencillamente a causa de la edad. Los anuncios de vacantes proclaman descaradamente, los límites máximos de edad para los solicitantes. De una u otra forma, las empresas ejercen políticas que alientan franca o encubiertamente, la discriminación laboral, por razón de la edad. Bajo toda consideración, la edad es un criterio demasiado arbitrario para servir de importante consideración aplicable a las técnicas de selección y de 
ascenso. (p. 1)

La edad es un factor discriminante en el caso de estudio colombiano, generándose así, la proyección a contar con un país que en los próximos años tendrá un alto porcentaje de personas con capacidad favorable para laboral en el campo del desempleo, desde lo cual se podrían generar brechas sociales que incrementen progresivamente la pobreza, viéndose además el Estado prever la atención de estas personas en razón de la salud y seguridad social, es una vertiente que abre el debate hacia la construcción de políticas públicas que contribuyan a minimizar la brecha que pueda originarse desde la posición de las empresas de no emplear a personas en edad productiva pero que por razones de discriminación son desechadas del campo laboral.

La discriminación laboral a causa de la edad, así como de otros factores, son violatorios de los derechos humanos fundamentales de las personas, una posibilidad de erradicar o minimizar el imaginario social impregnado sobre la discriminación laboral, es fomentar la formación ciudadana y empresarial sobre los derechos humanos desde una óptica multicultural, en este sentido, (Giménez e Isea, 2017, p 170) exponen que "partiendo de esto sustentamos la concepción de los derechos humanos como interculturalidad. Esta teoría busca que los derechos humanos se piensen desde una crítica multicultural dentro de la cual ellos se reformulen como instrumentos de emancipación”. Así mismo, otra violación de los derechos humanos y acto de discriminación laboral, es referida al sexo, en este sentido, (Ordóñez Yraolagoitia, 2015) plantea que

La discriminación en el trabajo por razón de sexo, es algo que, desgraciadamente, todos conocemos. Es innegable que este fenómeno se sigue produciendo, tanto en nuestro país como en nuestra comunidad autónoma, pero aunque conozcamos el problema que esto supone, ¿nos paramos a cambiarlo? Es una actitud que mucha gente tiene arraigada y que probablemente le salga de una manera inconsciente, ya que la discriminación en el trabajo es la última pieza de otra serie de actitudes machistas. (p. 262)

Los efectos de la discriminación por razón del sexo pueden producir no solo acciones de violencia xenofóbica, sino, generación de efectos negativos en las personas y 
grupos sociales que lo padecen, generando así, acciones negativas que afectan la autoestima de las personas afectadas, influyendo en la generación de relaciones interpersonales no asertivas a causa de acciones que lejos de contribuir en un clima de paz, generan perturbaciones aceleradoras de la violencia que termina por afectar tanto al emisor como receptor de la misma. Así mismo, (Horbath y Gracia, 2014), plantean que

Es fundamental reintroducir en la cuestión social la centralidad que tiene el trabajo para generar condiciones de mayor bien estar, justicia y equidad, y para coadyuvar a procesos de subjetivación que implique en el auto reconocimiento y la dignidad de las y los trabajadores. (p. 488).

Enfrentar la discriminación laboral, implica organizar movimientos sociales que se fundamenten desde la defensa de su dignidad humana, en establecer acciones jurídicas que permitan impulsar políticas públicas en función de la defensa y promoción del derecho al trabajo sin menoscabo del mismo, contribuyendo así en la edificación de una sociedad basada en la justicia y equidad social como factores que permitan contribuir en la generación de una sociedad con sustento en la paz, conciliación, respeto mutuo, con la finalidad de encontrar puntos de encuentros y no distanciarse por prácticas discriminatorias que afectan la dignidad de la persona. Sin embargo, desde la discriminación laboral podría generarse un movimiento en defensa de la dignidad humana, en este sentido, (Aldana e Isea, 2018) plantean que

Esta conflictividad si es asumida por las partes como un proceso dialéctico de crecimiento social, será beneficiosa en el largo plazo, dado que la sociedad crecerá mediante un aprendizaje reciproco. En cambio sí las partes se perciben como enemigos, es posible que los conflictos internos crezcan, germinando las bases del sistema axiológico social, lo cual sería en detrimento del bienestar colectivo, así como de una real defensa de los derechos humanos, por ende de la dignidad humana. (p. 20)

Es pertinente además fomentar un sistema deontológico social que permita despertar el interés en las personas por defender su dignidad y derecho al trabajo como un derecho universal, de ese modo, se podrá promover acciones movidas por la convicción de 
crecer mediante el trabajo como un medio que permite crecer como persona, como sociedad y transcender hacia la auto realización del ser humano en sus diversas dimensiones que le permitan percibirse realizado y no denigrado a causa del fenómeno discriminatorio laboral, el cual impide progresar individual y colectivamente hacia una sociedad de paz.

\section{Teoría de la dominancia social}

La teoría de la dominancia social procura explicar el modo como los mitos generados en la sociedad pueden conducir a la construcción de configuraciones individuales y colectivas sobre el rol dominante de un determinado extracto social sobre otros, en el caso de estudio de género, se evalúa como el hombre se mitifica en la sociedad por encima de la mujer. Esto permite considerar que en los imaginarios sociales se arraigan creencias sobre la fortaleza de un segmento poblacional, en este sentido, (Castillo Mayen, 2011), afirma que

Esta aproximación teórica defiende que las decisiones y conductas individuales, el surgimiento de nuevas prácticas sociales y el funcionamiento de las instituciones están determinados por mitos legitimadores, que consisten en valores, actitudes, creencias, estereotipos e ideologías culturales apoyados consensualmente. Dentro de estos mitos legitimadores se distinguen dos tipos, aquellos que promueven las jerarquías y aquellos que las debilitan. (p. 46)

En torno a la discriminación laboral, se evidencia la legitimidad establecida por los grupos dominantes en la generación de brechas como la edad, género, raza, religión, política, entre otros, como eslabones para descalificar a las personas laboralmente, discriminándola por pertenecer a un grupo minoritario, el cual en ocasiones es generado por la mitificación de las creencias populares sobre un determinado fenómeno social, afianzado así, los mitos discriminatorios que terminar por fragmentar la sociedad en sus bases morales y sociales. 


\section{METODOLOGÍA}

El tipo de investigación es documental con fines de "Analizar la discriminación laboral en razón del género y la edad en Colombia en el período comprendido entre enero de 2016 a junio de 2016", en este sentido, (Palella Stracuzzi y Martins Pestana, 2012, p. 90) señalan que esta investigación "Se concreta exclusivamente en la recopilación de información en diversas fuentes. Indaga sobre un tema en documentos escritos $u$ orales; uno de los ejemplos más típicos de este tipo de investigación son las obras de historia".

En cuanto al diseño de la investigación se refiere al bibliográfico, explicado por (Palella Stracuzzi y Martins Pestana, 2012) como aquel que,

Se fundamenta en la revisión sistemática, rigurosa y profunda de material documental de cualquier clase. Se procura el análisis de los fenómenos o el establecimiento de la relación entre dos o más variables. Cuando opta por este tipo de estudio, el investigador utiliza documentos; los recolecta, selecciona, analiza y presenta resultados coherentes. El diseño bibliográfico utiliza los procedimientos lógicos y mentales propios de toda investigación: análisis, síntesis, deducción, inducción, entre otros. (p. 87).

De ese modo, el investigador desarrolló un proceso investigativo en donde su población de estudio, se basó primordialmente en documentos escritos como leyes, normas, convenios, tesis de grado, investigaciones en revistas arbitradas, para escrutar tales manuscritos con el objeto de analizarlos y generar conclusiones que contribuyen a la generación de nuevos conocimientos en el campo de la discriminación laboral en Colombia.

En lo que respecta a las técnicas de interpretación se acudió a la técnica del análisis de contenido de la información recopilada, lo cual permitió formular resultados coherentes con el objetivo general de la investigación, generándose así, una discusión sobre el objeto de estudio con el fin de ampliarlo en el contexto del conocimiento del derecho laboral, además de visibilizar aspectos que son resaltantes en la consolidación del fenómeno discriminatorio en Colombia. 


\section{RESULTADOS}

Los resultados derivados de los hallazgos logrados indican que en general, en las empresas e instituciones de Colombia se practican o toleran discriminaciones respecto a las condiciones de la contratación, por razones de ciertas limitaciones por su género, por su condición femenina o masculina, no atribuyéndoles algunos trabajos por no encajar en el perfil correspondiente, no obstante a pesar de estar la persona aún capacitada para hacerlo.

De la misma manera, se ejecutan prácticas discriminatorias por razones etarias, o derivadas de alguna condición física que igualmente ha devenido por razón de la edad, pero que no les impide ejecutar algunas tareas distintas, imposibilitando en tal sentido la estabilidad laboral, o su antigüedad por algunos otros años, incluso no se establecen condiciones de trabajo adecuadas, ergonómicas, que pudieran permitir las labores convenientes a esos trabajadores que han alcanzado una edad específica.

Se evidencia que la praxis laboral colombiana en las organizaciones, sean públicas o privadas, crean perfiles de cargo atribuidos solo a hombres, como es el caso de gerencias, cargos ministeriales, cargos legislativos, puestos operativos de diversa índole, con lo cual se demuestra que aún la mujer sigue discriminada bajo el mito del sexo debil.

A pesar de las normas establecidas en la evolución del derecho laboral, todavía se habla de una escasa formación profesional para los miembros de la organización pública o privada, sobre todo en los tiempos actuales en los cuales la alta rotación de personal y los costos producidos para un aprendizaje organizacional efectivo, coartan cualquier iniciativa que pudiera devenir de empleadores emprendedores que pretendan producir el cambio organizacional.

Por otro lado, las organizaciones se circunscriben a emplear lo que salarialmente le reporte menos costos, sin tomar en cuenta la experiencia del solicitante a la vacante, su antigüedad en un cargo, o la creatividad y la iniciativa que puede derivar de un joven emprendedor; en tal sentido, las diversas ocupaciones son efectuadas por un personal 
que no muestra competencias para desempeñar el perfil del cargo formulado para el puesto de trabajo.

Así también, con relación a las adecuadas condiciones de trabajo que se exige en las organizaciones, en muchos casos se adolece de las mismas, porque la infraestructura no se encuentra normada según estandares de calidad y ergonomía, así como tampoco se preve elementos para la minimización de accidentes, necesarios a toda entidad empresarial.

A la vez, mujeres de un estatus económico bajo, con poca o ninguna educación, sufren abusos por motivo de género sobre todo cuando trabajan como empleadas domésticas $\mathrm{u}$ organizaciones donde fungen como secretarias de patronos que ejercitan el acoso laboral como práctica cotidiana.

Asimismo, este tipo de operadoras de escasos recursos, de niveles cognitivos bajos, son discriminadas también por su situación de maternidad, no teniendo acceso a la atención a la salud reproductiva porque el empleador no cuenta con seguros para ellas, debiendo acudir a centros hospitalarios que en mucho desdicen de su función de atención a los usuarios.

De la misma manera, se evidencia de algunas zonas o regiones colombianas, trabajadoras de ambos sectores enfrentados a la discriminación de género por parte de funcionarios del gobierno y empleadores que irrespetan a sus empleados por razones de poder, estabilidad económica o falta de cultura laboral. Asimismo, ocurre con aquellas poblaciones indígenas, donde la falta de formación es una condición proclive al maltrato, a las amenazas de despido, produciendo sensación de inestabilidad, de baja autoestima, de descalificación, como efectos devastadores de la discriminación.

La misma situación se ha evidenciado con respecto a jóvenes trabajadores, que son objeto de rechazo por su falta de experiencia, de repudio ante una labor mal ejecutada por su falta de experiencia en el área de trabajo, desconociéndoseles incluso el derecho reconocido nacionalmente a la jornada de ocho horas y la semana laboral de cuarenta y ocho horas, solo tienen derechos limitados a los días festivos y el descanso semanal, 
no disfrutan en su mayoría del derecho a la atención sanitaria para los trabajadores dentro del sistema nacional de seguridad social.

De igual forma, también se evidenció la trayectoria de las políticas públicas en Colombia, las cuales solo son dirigidas a grupos sectarios, dejando sin posibilidad de trabajo a personas experimentadas, con conocimiento, trayendo como consecuencia niveles de desempleo exorbitantes que han minado la calidad de vida de cientos de habitantes colombianos. También se evidenció de los hallazgos logrados, que si bien en Colombia se crean disposiciones que regulen el quehacer laboral de empresarios y empleadores en general, y normas en muchas ocasiones estas se convierten en letra muerta, dada la falta de reconocimiento al trabajo ocupacional, a la experiencia de los individuos, al respeto en general de su condición de seres humanos y de sus derechos más fundamentales.

De acuerdo a lo planteado, el investigador observa una especie de vacío o laguna de la ley, por cuanto las normativas no explicitan con verdadera eficacia, las sanciones ante su propio incumplimiento, por lo cual los empleadores continúan ejercitando políticas organizaciones desacordes con la legislación vigente. Se debe afirmar entonces la necesidad de la implementación en la praxis laboral colombiana del principio rector en materia de trabajo, de la dignificación humana, el cual resulta inoperante por cuanto se evidencia que se incurre en las arbitrariedades, desenfrenos y descuido de la ley, por parte de los empleadores, cuando se impide que los trabajadores obtengan la calidad de vida laboral con base en los beneficios derivados de su propia praxis laboral.

\section{CONCLUSIONES}

Con el desarrollo de este estudio es posible inferir que la discriminación laboral en Colombia por razón de género y edad, implica un trato diferenciador, negativo, hacia la situación de determinadas personas, que por razón de su condición de hombre o mujer, o su circunstancia etaria, no aplican para la ejecución de determinados trabajos. Estos fenómenos descritos evidencian que el desempeño actual del mercado laboral en 
Colombia no permite un salto cualitativo en términos del desarrollo económico y social del país.

En este sentido, ha de concluirse en primer orden pese a que ha habido un progreso en algunos indicadores de escogencia de sujetos que se adecuan al perfil de un cargo, tales como la experiencia y antigüedad, es necesario avanzar hacia políticas más ambiciosas de generación de ingresos en los hogares. Esto ayudaría a asegurar que los niños, niñas y adolescentes se dediquen principalmente, y de ser posible únicamente, a estudiar.

De allí que se estipule, como necesario, la adhesión en el sistema jurídico colombiano, de renovadas y modernas normas laborales, debiendo canalizar adecuadamente la prohibición de la discriminación por género y edad, bajo la supervisión de funcionarios públicos que penalicen la acción de empresarios o funcionarios públicos que impidan el trabajo de determinados sujetos, y con la imposición de sanciones que reivindiquen la exclusión perpetrada.

De la misma manera, tales normas deberán atender a la seguridad laboral de personas con condición de género y edad especial, además de continuar orientando medidas de medicina ocupacional en las empresas e instituciones públicas, de modo que se produzca la evitación de riesgos dentro de las políticas de gestión organizacional.

Asimismo, se deberá implementar políticas y medidas que diversifiquen el trabajo, de manera que se pueda producir el desarrollo armónico, socio-cultural de Colombia, con el consiguiente progreso del país, a través de la utilización de talentos que por su condición o edad hayan dejado de trabajar, o no hayan sido aceptados en las labores específicas donde se consideran expertos.

En igual sentido, se requiere aperturar agencias de empleos, públicas y privadas, nacionales y regionales, donde se analicen los patrones y criterios para ser definidos los perfiles de cargo; debiendo crear planes socio culturales de asistencia a personas que hayan sido excluidas del ámbito laboral, por alguna condición física, de edad, de manera que se produzca con ello la tutela del estado, la asistencia sanitaria y en 
materia de seguridad social, pudiendo con esto re-dignificar a algunos trabajadores excluidos.

\section{REFERENCIAS CONSULTADAS}

1. Aldana, Julio Juvenal y Isea, Josia. (2018). Derechos humanos y dignidad humana. Iustitia Socialis. Revista Arbitrada de Ciencias Jurídicas. Año III. Vol. III. NN4. Enero - $\quad$ Junio $2018 . \quad$ Disponible en http://fundacionkoinonia.com.ve/ojs/index.php/lustitia_Socialis/article/view/119.

2. Amaya Quimbaya, Mateo (2013). La Edad también es condición de discriminación para acceder a un empleo. Activo Legal 7 de mayo de 2013. Disponible en: http://www.activolegal.com/web/index.php/noticias/actualidad/466noticia070513n2.

3. Barraza Narváez, Nacira. (2010). Discriminación salarial y segregación laboral por género en las áreas metropolitanas de Barranquilla, Cartagena y Montería, de la Universidad del Norte de Barranquilla. Disponible en https://guayacan.uninorte.edu.co/publicaciones/upload/File/Documentos_IEEC_3 1.pdf.

4. Castillo Mayen, María del Rosario (2011). Discriminación de Género y Dominancia Social. Análisis de los Estereotipos de Género y de la Influencia del Priming Subliminal. Facultad de Humanidades y ciencias de la educación. Departamento de psicología. Tesis doctoral. Disponible en http://ruja.ujaen.es/bitstream/10953/318/1/9788484396017.pdf.

5. Castro Castro, José Francisco (2010). Discriminación en las relaciones laborales. Algunos casos particulares. Chile. Disponible en http://www.dt.gob.cl/portal/1626/articles-65173_recurso_1.pdf.

6. Diario Oficial No. 45.777 de diciembre 30 de 2004. LEY 931 DE 2004. Disponible en https://ustarizabogados.com/porvenir/images/leyes/LEY-931-DE-2004.pdf.

7. Giménez, Maribel e Isea, Josía. (2017). Justicia colectiva como mecanismo organizacional y reivindicativo de los derechos humanos en los privados de la libertad. Revista Arbitrada Interdisciplinaria Koinonía, v. 2, n. 3, p. 157-171. Disponible en: <http://fundacionkoinonia.com.ve/ojs/index.php/revistakoinonia/article/view/58>. 
8. Horbath, Jorge y Gracia, Amalia. (2014). Discriminación laboral y vulnerabilidad de las mujeres frente a la crisis mundial en México. Economía, Sociedad y Territorio, vol. xiv, núm. 45, 2014, 465-495. Disponible en http://www.scielo.org.mx/pdf/est/v14n45/v14n45a6.pdf.

9. Jiménez Lemus William. (2015). Discriminación laboral, por razón de la edad, en Colombia. Disponible en https://www.gestiopolis.com/discriminacion-laboral-porrazon-de-la-edad-en-colombia/.

10. Ordóñez Yraolagoitia, Leyre. (2015). Discriminación laboral por razón de género. Lan Harremanak/32 (2015-I) (262-277. Disponible en http://www.ehu.eus/ojs/index.php/Lan_Harremanak/article/view/15417.

11. Organización Internacional del Trabajo (1960). Convenio relativo a la discriminación en materia de empleo y ocupación Disponible en https://www.ilo.org/dyn/normlex/es/f?p=NORMLEXPUB:12100:0::NO::P12100_IL O_CODE:C111

12. Organización Internacional del Trabajo (2014). Guía sobre las normas internacionales del trabajo. Disponible en https://www.ilo.org/wcmsp5/groups/public/---ed_norm/--normes/documents/publication/wcms_246945.pdf.

13.Palella Stracuzzi, Santa y Martins Pestana, Feliberto. (2012). Metodología de la investigación cuantitativa. Fondo editorial de la Universidad Pedagógica Libertador. Caracas, Venezuela.

14. Vargas Mendieta, Miller Jaime y Cortes, Mariluz (2011). La edad como una barrera laboral, un análisis descriptivo por genero a la PEA de 40 a 60 años de edad en Colombia 2004-2008. Tesis Universidad de la Salle, Colombia. Disponible http://repository.lasalle.edu.co/bitstream/handle/10185/12490/T10.11\%20V426e. pdf? sequence $=1$

C2019 por el autor. Este artículo es de acceso abierto y distribuido según los términos y condiciones de la licencia Creative Commons Attribution (http://creativecommons.org/licenses/by/4.0/). 